\title{
The Semi-Physical Simulation System Designed for Space Optical Remote Sensing Based on 6-DOF Motion Platform
}

\author{
Ming Xuan $\mathrm{Li}^{1, \text { a }}$, Gang Wu Jiang ${ }^{2, \mathrm{~b}}$ and Rui Zhang ${ }^{3, \mathrm{c}}$ \\ ${ }^{1}$ Information Engineering University, Zhengzhou, China \\ ${ }^{2}$ Information Engineering University, Zhengzhou, China \\ ${ }^{3}$ Information Engineering University, Zhengzhou, China \\ aImxuan0529@163.com, bjianggw@163.com, cjeffor@163.com
}

\begin{abstract}
Keywords: Space Optical Remote Sensing Imaging technology, TDI CCD, 6-DOF Motion Platform, Semi-Physical Simulation.

Abstract. With the development of space optical remote sensing imaging technology, Space remote sensing systems have reached a new stage which is changing towards higher spatial resolution, higher spectral resolution, higher radiometric resolution, and higher temporal resolution. In recent years, applying TDI CCD to earth observation from space has become a growing trend, and it can make integrals to the same moving target by using multilevel sensitive units, which has improved the $\mathrm{S} / \mathrm{N}$ and the dynamic range of optical system effectively. This paper proposes a semi-physical simulation system designed for space optical remote sensing based on 6-DOF motion platform. The system ignores the atmospheric radiation and it can obtain high quality images by simulating the whole physical process of the space optical remote sensing imaging chain which includes the sensor of TDI CCD, the motion platform of satellite, the ground scene, and the access to final remote sensing.
\end{abstract}

\section{Introduction}

Nowadays, remote sensing technology has become a necessary Hi-tech means of acquiring geographical environment and monitoring change detection ${ }^{[1]}$. Optical remote sensing imaging technology is not only an important branch of remote sensing, but also a significant component of improving space science technology. The limit of observation condition make it difficult to transmit remote sensing data under various circumstance, and the cost of aerospace flight test and space satellite test are much expensive. Consequently the quality of the image cannot satisfy the requirement of remote sensing detection ${ }^{[2]}$. So many scholars attempt to simulate the process of space remote sensing imaging under the laboratory condition, which can take place of complex and expensive tests of aerospace flight and space satellite. The simulation is regarded as a valid and perspective method of test. Because the process of space remote sensing imaging system is very complicated, many parts of the system are unable to be described completely and accurately through mathematic model. However, the semi-physical simulation technology can take place of corresponding mathematic model by putting physical object into the simulation system as much as possible, which is closer to practical situation ${ }^{[3]}$. Besides saving cost, the semi-physical simulation can avoid the difficulty of modeling and the error simulation brought by modeling, as well as displaying the process of remote sensing accurately and fully. So it is meaningful to establish the semi-physical simulation system designed for space optical sensing under laboratory condition.

\section{The space optical remote sensing imaging chain}

The whole system of space optical remote sensing include three parts. First, the information of ground scene which is reflected or radiated by the sun transfers to the satellite platform's sensor over atmosphere, and turns into imagery information. Second, after photoelectric conversion, the message is transferred to ground of the earth. Finally, the message can represent the information of ground scene on the display device. 
The process of optical remote sensing imaging. The chain of the process usually consists of the ground scene, illumination source, atmosphere, satellite platform, remote sensor, data processing and transmission, data receiving and processing on the ground, image interpretation, the users and so on ${ }^{[4]}$. Figure. 1 shows the sketch of optical remote sensing imaging procedure, Figure. 2 shows the block diagram of optical remote sensing imaging procedure.

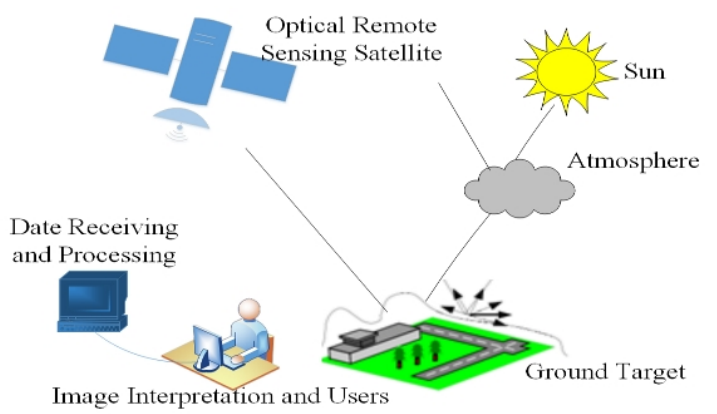

Figure. 1. The sketch of procedure

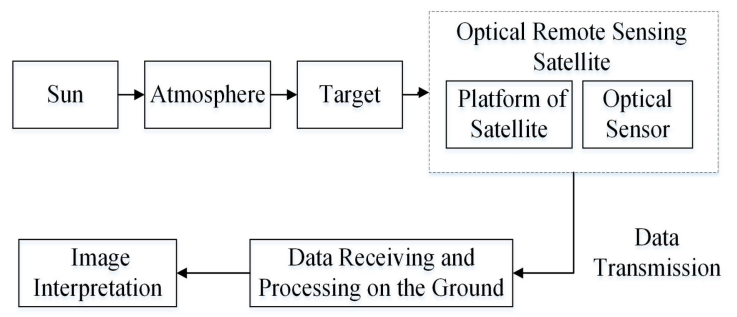

Figure. 2. The block diagram of procedure

In space optical imaging remote sensing, the sun is used as a lighting source. When solar radiation passes through the earth's atmosphere to reach the target, the atmosphere absorbs and scatters solar radiation, parts of solar radiation on the target are reflected. When the solar radiation of target's reflection through the atmosphere before reaching the satellite, the atmosphere absorbs and scatters solar radiation again. Optical remote sensing satellites are used for detection of electromagnetic radiation from the target to itself. Solar radiation is detected by optical remote sensors, and it not only includes the solar radiation that target's directly reflection, but also includes atmospheric scattering solar radiation. The optical system of the optical remote sensor will focus the collected electromagnetic radiation on the detector, then the detector converts it to electrical signal. Electrical signal is amplified, filtered and digital processed by the signal processing circuit and then will be sent to ground station by data transmission system. The remote sensing data received by ground station is recorded on a storage medium, then the original remote sensing image has been obtained. The pretreated remote sensing data can usually be shown on a computer monitor, and also can be further processed according to different requirements, such as edge enhancement, image registration, and contrast extension, etc. The processed remote sensing image data, can be drilled by remote sensing informative model. So we can get the remote sensing information needed, such as supervised classification and unsupervised classification ${ }^{[5,6]}$. In general, we can obtain various interesting targets.

The imaging principle of TDI CCD. TDI CCD is a new type of CCD, it has plane array structure and linear array output. Compared with the common linear CCD, TDI CCD has the function of multiple series delay integral. Judging from its structure, the number of columns are the line of pixel elements of it, and the number of rows are the series of it. Generally the available series for it can be 16 , 32,64 , and 96 to the maximum value 256. TDI CCD is mainly adopted by earth imaging, which is exposed on the same target many times, thus extending optical integration time, increasing the solar energy collection, and significantly improving signal strength.

To ensure the quality of the image, according to the special working principle of TDI CCD, it require that the same target should be exposed and integrated by each pixel on the same column. That is to say, the TDI CCD line scan rate and the velocity of the moving platform should be strictly synchronized ${ }^{[7]}$. According to the principle of TDI CCD imaging, formula is as follows. $T_{\text {int }}$ is the integration time of satellite orbit operating, $d$ is the focal length of the camera, $f$ is the pixel size of TDI CCD, $V_{T}$ is the velocity of the camera relative to target scene, $H$ is the distance between the camera and the target scene. 


$$
T_{\text {int }}=\frac{d / f}{V_{T} / H}
$$

So we can see that when the focal length and pixel size are fixed, the camera's line scan rate is related to the velocity of the camera relative to target scene and the distance between the camera and the target scene.

\section{The program of semi-physical simulation}

For the entire process of space optical remote sensing imaging and the various studies on the semi-physical simulation ${ }^{[8]}$ and the 6-DOF motion platform ${ }^{[9,10]}$, this paper proposes a semi-physical simulation program of space optical remote sensing based-on 6-DOF motion platform. The block diagram of semi-physical simulation program is shown in Figure. 3.

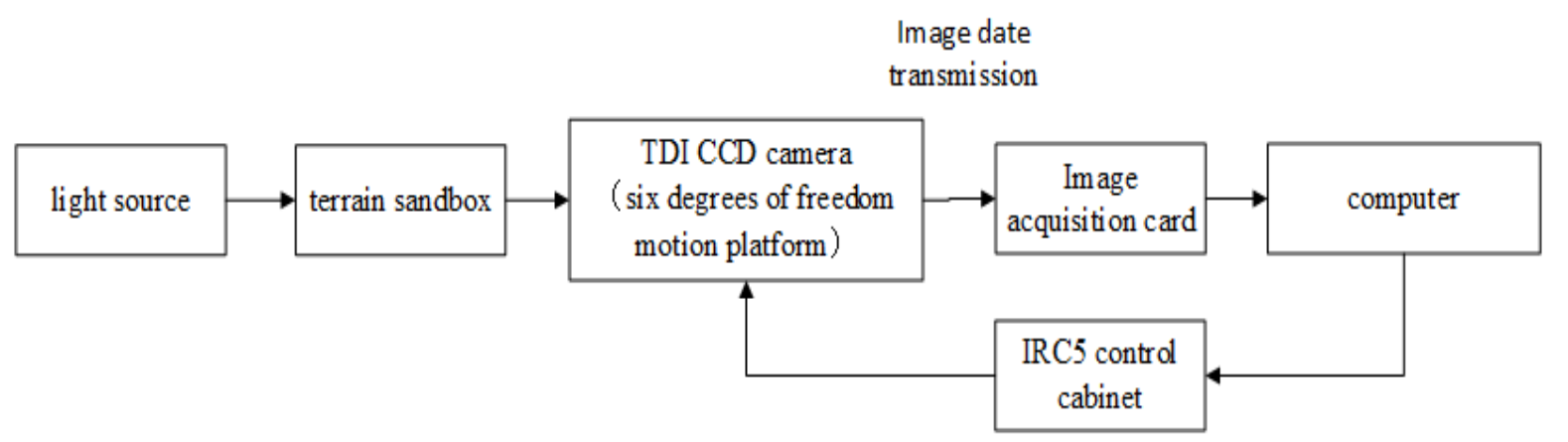

Figure. 3. The block diagram of semi-physical simulation program

The main equipment of simulation: terrain sandbox, adjustable light source, linear array TDI CCD camera, six degrees of freedom motion platform, computer, etc. The system consists of five modules: light source module, sandbox terrain environment module, optical remote sensing satellite module, data transmission module, and data display module. Due to the limitation of physical model, the whole space optical remote sensing imaging system has been simplified. The system does not consider the link that atmosphere to the sun light's reflection and refraction, and the data transmission module is replaced directly by the camera cable connected with computer storage medium.The biggest innovation of the whole system is taking six degrees of freedom motion platform as a linear array of TDI CCD camera motion platform. By setting the motion patterns of six DOF motion platform, we can get clear TDI CCD imaging, thus simulating the process of space remote sensing imaging for earth observation. The sketch of semi-physical simulation program is shown in Figure. 4.
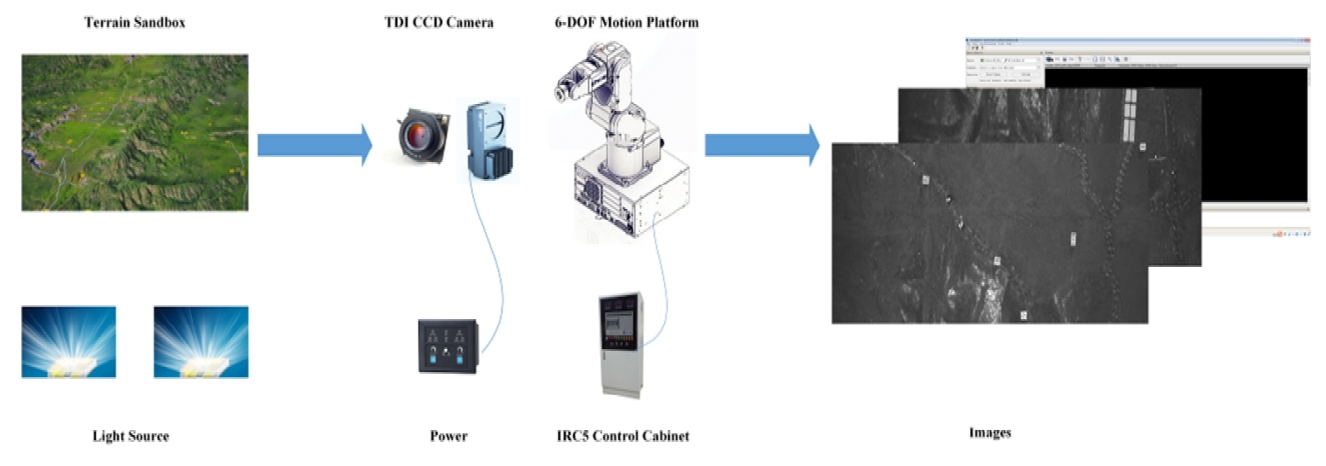

Figure. 4. The sketch of semi-physical simulation program

\section{The program of six degrees of freedom motion platform}

The moving speed and stability of the six DOF motion platform directly determine whether the quality of final simulative image is clear or not. This paper uses the industrial robot-IRB2600ID of ABB 
company, which has six degrees of freedom. IRB2600ID is shown in Figure. 5, and the main technical parameters of it is shown in Table 1.

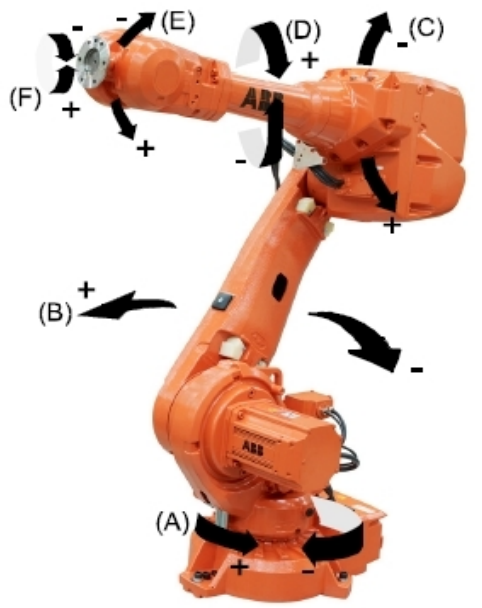

Table 1. The main technical parameters of IRB2600ID

\begin{tabular}{|c|c|}
\hline Mechanical structure & technical parameters \\
\hline Weight $[\mathrm{Kg}]$ & $273-276$ \\
\hline Height $[\mathrm{mm}]$ & 1600 \\
\hline Supply voltage $[\mathrm{V}]$ & 380 \\
\hline Maximun load $[\mathrm{Kg}]$ & 15 \\
\hline Location accuracy $[\mathrm{mm}]$ & $0.023-0.026$ \\
\hline Path accuracy $[\mathrm{mm}]$ & $0.27-0.30$ \\
\hline
\end{tabular}

Figure. 5. IRB2600ID

The controlling strategy of 6-DOF motion platform. IRB2600ID is controlled by IRC5 control cabinet, and there are usually two kinds of control mode, as shown. One is through the communication between the handle and IRC5 controller, which can be divided into manual control and RAPID programming control. Another is through the communication between the computer with IRC5 control cabinet, which uses the software of RobotStudio through RAPID on-line programming to control the 6-DOF motion platform. In essential both two modes above are through RAPID instructions to control the 6-DOF motion platform. It can realize many kinds of sports, such as linear translational motion, arc movement, single axis rotation movement and so on. The speed of the robot also can be programmed to be controlled, range in 10-2000 mm/s. According to the scope of sandbox size, linear translation speed is set as $100 \mathrm{~mm} / \mathrm{s}$. By the formula (1), if we want to get clear images, the TDI CCD line scan rate and the velocity of platform must be synchronized strictly, so the line frequency for TDI CCD camera is will be set correspondingly. TDI CCD is carried on the sixth axis of platform. According to the above plan, six DOF platform is controlled from one position to another position in a linear way to scan the terrain sandbox, then we can get clear linear array images. The linear images of terrain sandbox are shown in Figure. 6.
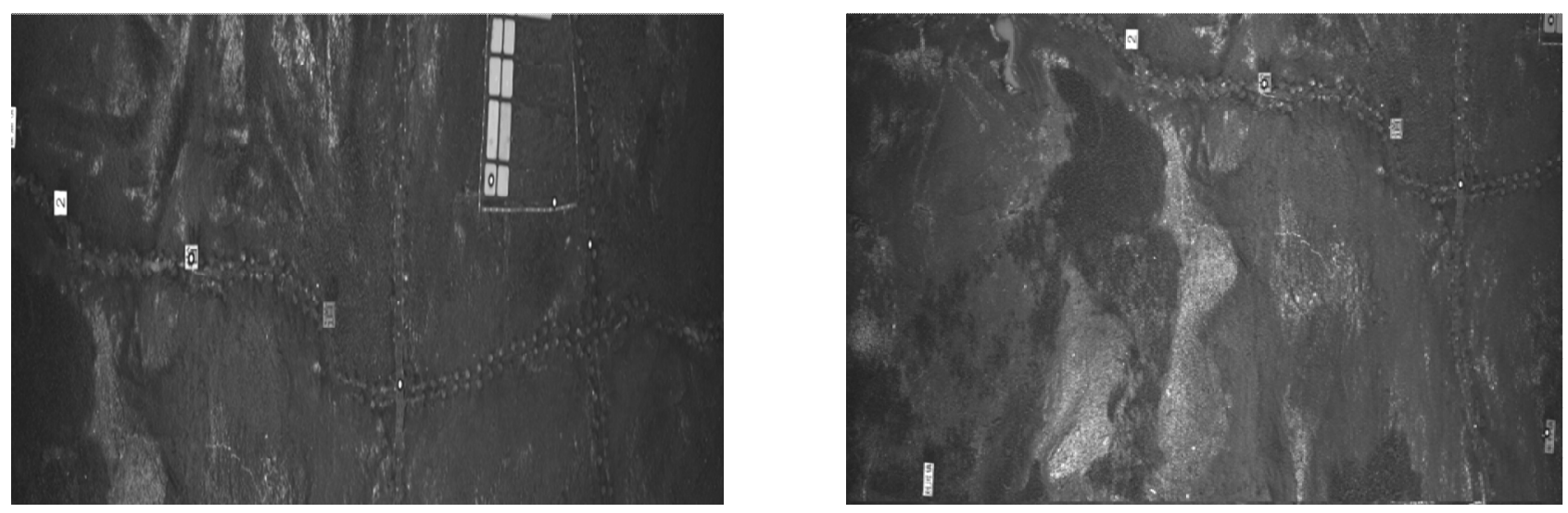

Figure. 6. The linear images of terrain sandbox

\section{Conclusions}

This paper first analyzes the specific process of space optical remote sensing imaging chain, and studies the imaging principle of TDI CCD which is the most potential sensor currently. On the basis of the 
features of six DOF motion platform, the paper puts forward the semi-physical simulation system which is based on six degrees of freedom motion platform in the first time. By adjusting the light source brightness, camera line frequency, and the speed of the platform, the system can get clear terrain image of sandbox, achieving the expected effect of simulation.

This semi-physical simulation system also can undertake a series of research work in remote sensing field, includes: the orbit and attitude of on-orbit space remote sensing satellites for a real-time simulation, ground targets tracking by remote sensing satellite simulation, Spacecraft rendezvous and docking simulation, camera imaging simulation under different spectrum light sources, various flags of close range photogrammetry imaging simulation under different brightness of light sources, a variety of different aperture of cameras imaging simulation, etc. The image obtained by this system can be used for quality evaluation of imaging, image matching and splicing, spacecraft cooperative target recognition, etc. So the proposed semi-physical system has a great value for research and application in the field of remote sensing, which will improve the level of research in space remote sensing science and technology in China.

\section{References}

[1] Remote Sensing Engineer Manual, US Army Corps of Engineers, 2003.

[2] Fan Bu. Study on Modeling and Simulation of Optical Remote Sensing System and Image Processing Technology [D]. Graduate school of Chinese academy of sciences (Institute of Optics and Precise Mechanics in Xi'an), 2014. (In Chinese)

[3] Sheng Xue, Xuekui Liu, Zhaowei Sun. The semi-physical simulation system design of micro-satellite based-on HLA. International Conference on Advances in Computational Modeling and Simulation. Procedia Engineering 31(2012) 801-806.

[4] Wenpo Ma. Aerospace Optical Remote Sensing Technology. Beijing: Chinese Science and Technology Press, 2010. (In Chinese)

[5] Liu Zhang, Zhiyuan Sun, Guang Jin. Design of Physical Simulation System for TDI CCD Dynamic Imaging [J].Optical Precision Engineer. 03:641-650, 2011. (In Chinese)

[6] Yingying Gu. The Research of Image Terminal Quality Assesment for Spaceborne Optical Remote Sensor[D]. Graduate School of Chinese Academy of Sciences (Institute of optical fine mechanics and physis in changchun.2013. (In Chinese)

[7] Xiubin Yang, Xing Zhong, Guang Jin, Liu Zhang, Zhiyuan Sun. Influence of the TDI CCD camera takes pictures when high resolution satellite lateral swaying. In Mechatronics and Automation, 2009. ICMA 2009. International Conference on , pp.1899-1903, 9-12 Aug 2009.

[8] Sheng Liu, Bowen Xing, Fei Peng, Bing Li. Design of semi-physical simulation platform for ship cooperative control system[R]. Intelligent Control and Automation (WCICA), 2014 11th World Congress on.

[9] An-yi Huang, Wen Cui, Heng nian Li. Semi-physical simulation system of autonomous optical navigation for Mars-approaching phase. In Guidance, Navigation and Control Conference (CGNCC), 2014 IEEE Chinese , pp.1409-1414, 8-10 Aug 2014.

[10] Boge T, Ou Ma. Using advanced industrial robotics for spacecraft Rendezvous and Docking simulation. In Robotics and Automation (ICRA), 2011 IEEE International Conference on , pp.1-4, 9-13 May 2011. 